\title{
Investigations into packaging technology for membrane-based thermal flow sensors
}

\author{
G. Dumstorff, E. Brauns, and W. Lang \\ Institute of Microsensors, -actuators, and -systems (IMSAS), Microsystems Center Bremen, University of \\ Bremen, Bremen, Germany \\ Correspondence to: G. Dumstorff (gdumstorff@imsas.uni-bremen.de)
}

Received: 3 September 2014 - Revised: 8 January 2015 - Accepted: 8 January 2015 - Published: 5 February 2015

\begin{abstract}
A new packaging method to mount a membrane-based thermal flow sensor, flush with the surface, is presented. Therefore, a specific design for the housing is shown, which is also adaptable to other conditions. It has been experimentally shown that it is important to mount the sensor flush with the surface. In addition, the experimental results are discussed. If a membrane-based thermal flow sensor is not mounted flush with the surface, vortices can occur (depending on velocity and fluid properties) or the reduction in the channel cross section plus a decrease in sensitivity have to be taken into account.
\end{abstract}

\section{Introduction}

Over the last decades, many different thermal flow sensors have been developed for the accurate measurement of gas flows (Van Putten and Middelhoek, 1974; Van Herwaarden and Sarro, 1986; Johnson and Higashi, 1987; Ashauer et al., 1999; Mailly et al., 2001; Ito et al., 2010; Adamec and Thiel, 2010; Ma et al., 2009). A wide range of flow sensors have been developed for different applications, such as automotive, industrial, and medical. Commercial flow measurement systems using MEMS (micro-electro-mechanical systems) devices are also available (sen, http://www.sensirion. com/; axe, http://www.axetris.com/; ist, http://www.ist-ag. com/; hon, https://www.honeywellprocess.com).

Besides sensor fabrication, the packaging technology is equally essential for building a flow measurement system. Here are some reasons underpinning the importance of flow sensor packaging.

- The chip has to be placed in a fluidic channel. Turbulence caused by, e.g., sharp edges of the chip, should be avoided. Eberhardt et al. (2003) showed that there is a point of discontinuity in the characteristic curve, because of turbulence at the edge of the flow sensor (Eberhardt et al., 2003). Due to a misalignment of the flow sensor in the channel, the sensitivity can significantly decrease.
- The membrane should be pressure equalized. If not, the membrane may be deflected and the characteristic curve may change. In addition, the heater resistance increases because of the piezoresistive effect. This can have an influence on the heater regulation.

- The flow system has to be sealed. Otherwise, the measurement results will be biased.

For the building up of a flow measurement system, different approaches have been developed over the years. A common approach relies on a monolithically integrated channel implemented at wafer level. Different types of these channels have been developed in the past years (Liu et al., 2012; Dijkstra et al., 2008; Buchner et al., 2007; Billat et al., 2008). The main disadvantages of such monolithically integrated microchannels are the need for an expensive wafer area, less material choice, and the fact that, due to the microchannels, large flow rates can only be measured by means of the bypass technique. In addition, packaging is still necessary to connect the tiny fluidic inlets and outlets, which could be a tricky task. Another approach to building a sensor system is in the postprocessing, where a flow sensor is integrated into a housing. This is what we focus on in this paper. In most publications, post-processing is only superficially treated, and sensor design, technology and performance are discussed in detail (Dillner et al., 1997; Sabaté et al., 2004; Bruschi et al., 2005). Different concepts for a packaging technology have already 


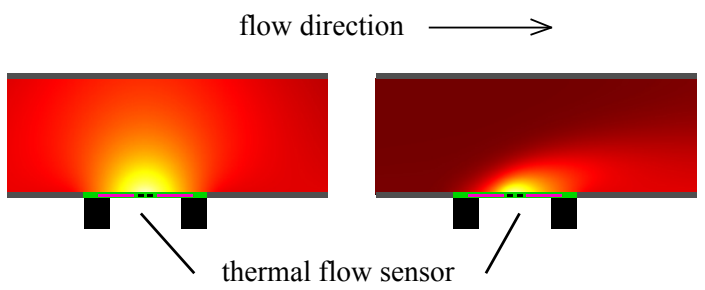

Figure 1. Illustration of the thermal field of a flow sensor. Left: $\dot{V}=0$; right: $\dot{V} \neq 0$

been presented, e.g., by Bruschi et al. (2009) or Kaltsas et al. (2002), but detailed information about housing design and the packaging process can not be found. Furthermore, there are no investigations on how misarrangement of the sensor can affect the characteristic curve. Of course, no detailed information on commercial flow measurement systems like sen, axe, ist and hon can be found (see the Introduction, first paragraph, p. 1).

For these reasons, we will first focus on the technology of packaging and housing for a precise assembly of a flow sensor chip in a channel. Afterwards we will demonstrate and discuss the importance of mounting the flow sensor flush with the surface. Therefore, we assembled flow sensors in a channel with a known misalignment.

\section{IMSAS flow sensor}

\subsection{Theory}

The IMSAS flow sensor (Fig. 2) is based on the calorimetric principle. It consists of a silicon nitride membrane with two thermal sensing elements and a heater in between. A membrane is essential for a high-performance thermal flow sensor because of the thermal decoupling of the functional elements. This in combination with a small thermal mass is the reason for a high sensor performance. When the heater is switched on and the volume flow $\dot{V}$ is equal to zero, a symmetric thermal field is generated in the flow channel (see Fig. 1, left). The temperature difference between the thermal sensing elements is zero. By applying a pressure difference between the channel inlet and the channel outlet, the thermal field becomes asymmetric and a temperature difference is measured (see Fig. 1, right). The temperature measurement is done by thermopiles based on the SEEBECK effect. While the hot junction of the thermopile is placed at a defined distance $d_{\mathrm{HT}}$ from the heater, the cold junction is placed on the bulk silicon, which acts as a heat sink due to its high thermal conductivity. A mathematical model of the IMSAS flow sensor can be found in Sosna et al. (2008). To get higher output voltages, several thermopiles are connected in series.

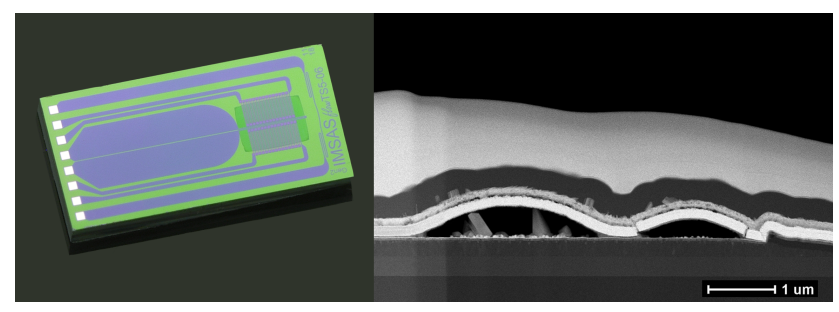

Figure 2. Left: the IMSAS flow sensor; right: the tungsten nitride layer is spalling because of stress and thermal shock in the LPCVD passivation process.

\subsection{Fabrication}

An IMSAS flow sensor and its fabrication were first presented in Buchner et al. (2006). The base of this sensor is a $600 \mathrm{~nm}$ thick membrane $(800 \mu \mathrm{m}$ long and $600 \mu \mathrm{m}$ wide in the flow direction) made of low-stress silicon-rich LPCVDSilicon-Nitride (LPCVD-SiN). All functional structures are passivated by this silicon nitride membrane. The thermocouples are made of boron-doped poly-silicon and tungstentitanium as well as a heater and a thermistor. The SEEBECK coefficient of one thermopile is $0.24 \mathrm{mV} \mathrm{K}^{-1} ; 15$ thermopiles are connected in series. Connecting thermopiles in series has an impact on the accuracy: while the output voltage increases with $n$ (the number of thermopiles), the Nyquist noise increases with $\sqrt{n}$. The single chips have a length of $3.5 \mathrm{~mm}$ and a width of $1.8 \mathrm{~mm}$ and consist of eight bond pads. Over the past years, new developments have been introduced into the process flow. The most important innovation is a hightemperature stable diffusion barrier at the junction between the metal and the semiconductor, made of tungsten nitride. Without the tungsten nitride layer, it would not be possible to passivate the functional structures in the LPCVD process because diffusion would destroy the junction, which can be seen in Fig. 2. The fabrication processes are described and illustrated in Fig. 3.

\section{Packaging technology}

\subsection{Housing}

The housing, shown in Fig. 4, consists of two parts: A is the base part and B is the flow channel part. In the base part, a $500 \mu \mathrm{m}$ deep rectangular cavity (A1, Fig. 4) is integrated to accommodate the flow sensor (C, Fig. 4). The cavity is made $400 \mu \mathrm{m}$ wider than the sensor. No influence on the flow, e.g., turbulence, is expected. A further cylindrical cavity $(500 \mu \mathrm{m}$ deep, $1 \mu \mathrm{m}$ in diameter) is placed inside the rectangular cavity in the membrane position (A2, Fig. 4). This cylindrical cavity is important for the pressure equalization. It should prevent adhesive flows through capillary forces under the membrane from sealing the back volume of the flow sensor. Of course, due to this setup, there is a bypass under the membrane. From simulations of the geometry, it was found that 


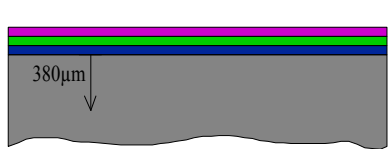

- Deposition of 500nm oxide, 300nm LPCVD low stress nitride and $300 \mathrm{~nm}$ in situ boron-doped poly-silicon

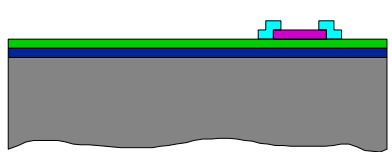

- Etching of poly silicon in RIE process + nitric acid - Deposition of $80 \mathrm{~nm}$ silicon oxide and structurization in RIE process

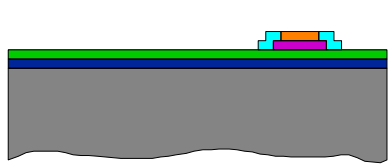

- Deposition of $60 \mathrm{~nm}$ titanium nitride (diffusion barrier) and etching with RIE-Chlorine process

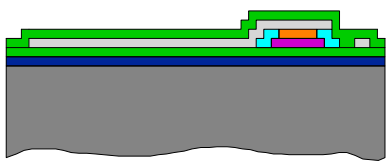

- Deposition of 200nm tungsten titanium and etching with WTi-Etch plus

- Deposition of 300nm LPCVD low stress nitride at $800^{\circ} \mathrm{C}$

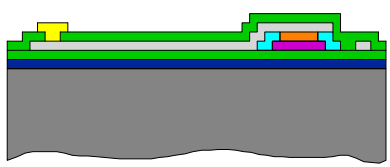

- Etching of nitride in DRIE process - Sputtering of 300nm gold for electrical connection

- Wet etching of gold with iodine

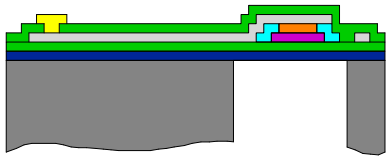

- Etching the bulk silicon in DRIE process and stoping on the oxide

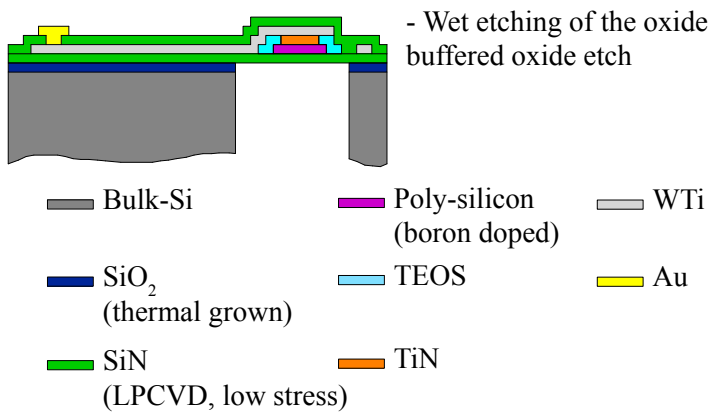

Figure 3. Process flow of the IMSAS flow sensor.

the flow thorough the bypass is less than $0.2 \%$ at $1 \mathrm{sLm}$ for air as fluid. Furthermore, the base part has an accommodation for a PCB board, which can be placed next to the base part for electrical connection by wire bonding. The hollow is designed in such a way that the PCB board is mounted flush with the surface (D, Fig. 4). The flow channel part contains the flow channel, whose geometry can be adapted by varying the cross section. In our setup, we set the width of the channel to $a=1.5 \mathrm{~mm}$ and the channel height to $1.75 \mathrm{~mm}$. It should be mentioned that the possibilities of changing the width of the channel are limited because of the dimensions of the sensor. The membrane should be placed in the middle
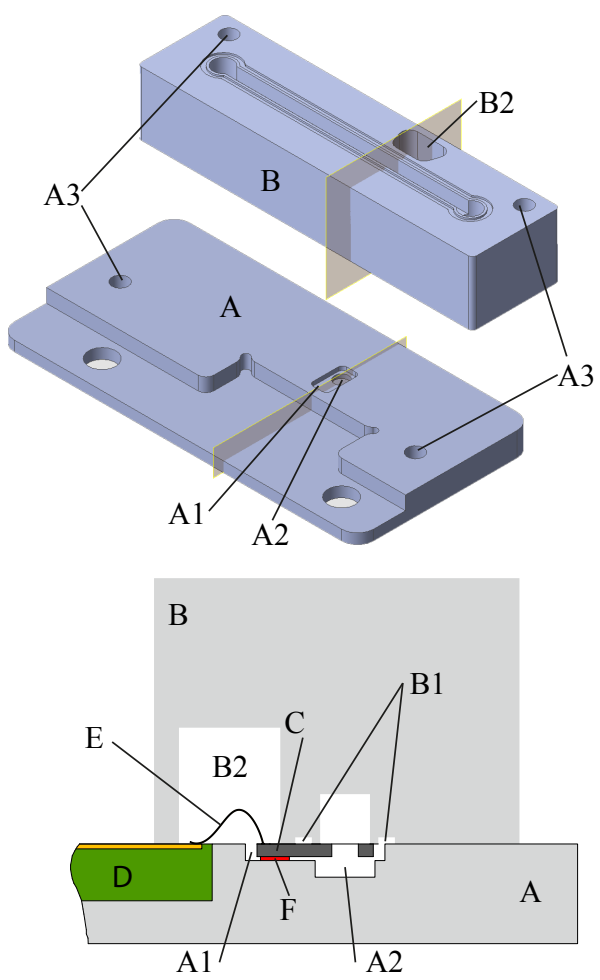

Figure 4. Top: CAD drawing of the base part (top view) and flow channel part (bottom view); bottom: cross section through the assembled parts referring to the plane in the CAD drawing. A = base part, $\mathrm{A} 1=$ cavity for the sensor, $\mathrm{A} 2=$ cavity for pressure equalization, $\mathrm{A} 3=$ drill holes for assembling both parts with aligning pins, $\mathrm{B}=$ flow channel part, $\mathrm{B} 1=$ capillary stop for adhesive, $\mathrm{B} 2=$ cavity to protect bond wires, $\mathrm{C}=$ sensor, $\mathrm{D}=\mathrm{PCB}, \mathrm{E}=$ bond wire, and $\mathrm{F}=$ adhesive

of the flow channel. Otherwise, there is a reduction in sensitivity. The flow channel is surrounded by a smaller channel integrated as a capillary stop for the adhesive when the flow channel part and the base part are fixed (B1, Fig. 4). To protect the bond wires, a cavity is built into the flow channel part (B2, Fig. 4). If electrical conductive fluids are used, the bond wires have to be sealed by a glob top. The measurements in this paper are only done with air; thus, we did not use any protection for the bond wires. Two drill holes in the base and flow channel part (A3, Fig. 4) are integrated for assembling both parts with aligning pins. Fabrication was done by milling the parts out of polycarbonate because tests were only performed with air.

\subsection{Sensor integration in the housing}

Sensor integration in the base part is done with a microsystem assembling machine from finetech. It is especially made for flip chip processes, and it consists of several tools, e.g., for the targeted inclusion of chips in a housing. The principle structure of the machine can be seen in Fig. 5. To integrate the flow sensor into the cavity of the base part, a thermode 
was developed (see Fig. 5). It can be placed in a special mechanical receiver integrated into the machine. The thermode must satisfy two requirements:

- picking the sensor without destroying the membrane; and

- mounting the sensor in the base part flush with the surface to avoid sharp edges provoking turbulence.

To satisfy the first requirement, the thermode picks the sensor outside of the membrane. Through a hole in the thermode, the sensor can be held by a vacuum. For the second requirement, the thermode is wider than the cavity. When the sensor is placed in the cavity, the thermode rests on the surface (Fig. 6d). To cure the adhesive, the thermode can be heated by a temperature controller.

The process of sensor integration is illustrated in Fig. 6 . In the beginning of the process, the base part is put on the swivel table. Using the dispensing system, a drop of a fast curing adhesive (DELOMONOPOX ${ }^{\circledR}$ NU355) is placed in its cavity. Then, the sensor is picked by the thermode (Fig. 6a). Curing the adhesive thermode with the sensor is aligned with the cavity of the base part. As seen in Fig. 6c, the top camera with the beam splitter shows the sensor held by the thermode and the base part with the cavity where the drop of adhesive lies. By pivoting the swivel head forward, the sensor is placed in the cavity flush with the surface of the base part (Fig. 6d). The thermode is heated to $T=140^{\circ} \mathrm{C}$ and the adhesive is cured in a few seconds. The vacuum for holding the sensor is switched off and the thermode is lifted up.

To contact the flow sensor, Al bond wire with a wedgewedge process was used (see Fig. 6). The bond wires are not sealed and thus it is only possible to measure air or other electrical non-conductive fluids. If water or other fluids are used, the bond wires have to be protected by a glob top.

In the last step, the base part and the flow channel part are combined and fixed with a common two-component adhesive. By optical inspection it was found out that the capillary stop in the flow channel part worked very well: the epoxy resin stopped exactly at the capillary stop. For flow connection, two fittings are screwed into the flow channel part: at the inlet and the outlet (see Fig. 7).

\section{Characterization}

\subsection{Flow measurement}

As flow references, MKS 1179 mass flow controllers (pressure at inlet 2 bar, resolution $0.002 \mathrm{sLm}$ ) have been used. The data acquisition from the sensor has been carried out with a USB-6212 card from National Instruments in combination with LabView. A sampling rate for data acquisition of $1 \mathrm{kHz}$ has been chosen. To control the heater, three different principles can be used:

- constant voltage;

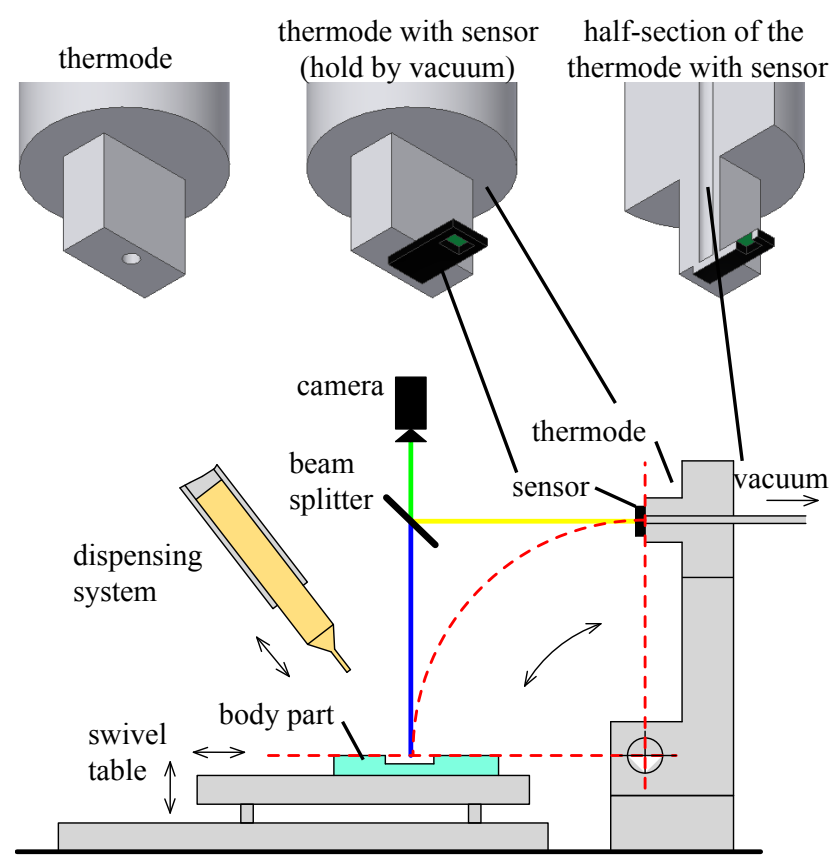

Figure 5. Principle illustration of the FINEPLACER ${ }^{\circledR}$ from finetech.

- constant temperature; and

- constant power.

Controlling systems for constant power and constant temperature can be found in Sosna et al. (2010) and Sturm et al. (2012). For our measurements, we used a constant voltage of $3.2 \mathrm{~V}$ for the heater. The deviation of the heater resistance in our systems was less than $1 \%$. Thus, the temperature of the heater at $3.2 \mathrm{~V}$ is nearly the same in all systems, and the measurements are comparable. We also compared constant voltage operation with constant power operation - however, we could not see any difference between the modes, because the resistance of the heater varies only slightly with the temperature.

\subsection{Influence of the sensor on the step height}

A flow sensor can be mounted in a channel in three different ways illustrated in Fig. 8: no step, step up, and step down. To analyze the influence of the step height on the characteristic curve, sensor systems of these three different types were built. Mounting the sensor step up was done with the help of a $100 \mu \mathrm{m}$ thick stainless steel sheet with a hole (according to the geometry of the cavity) that was laid on the body part, and the sensor was then mounted as described in Sect. 3. The sensor mounted step down was fixed with adhesive on the bottom of the sensor cavity (see A1 in Fig. 4). Both step heights were around $100 \pm 2 \mu \mathrm{m}$, which was tactile measured (Ambios XP-2). Of course, the system with no step was mounted in the way described in Sect. 3. The result 

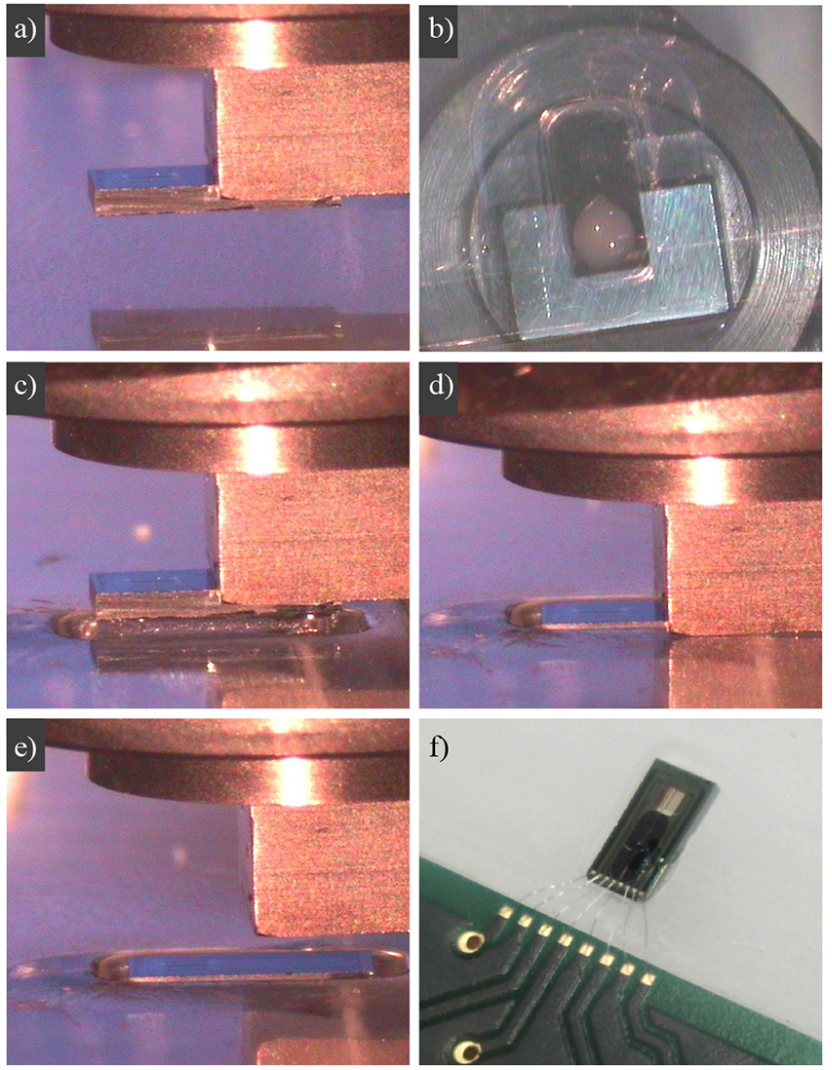

Figure 6. Flush mounting of the sensor in the base part. (a) Picking of the sensor outside of the membrane (b) because of the camera with a beam splitter sensor and cavity can been seen and aligned; (c) the sensor is set in the cavity; (d) the thermode is heated; (e) the thermode is lifted, and the sensor is mounted flush with the surface; (f) IMSAS flow sensor integrated into the base part and electrically connected by $\mathrm{Al}$ bond wires.

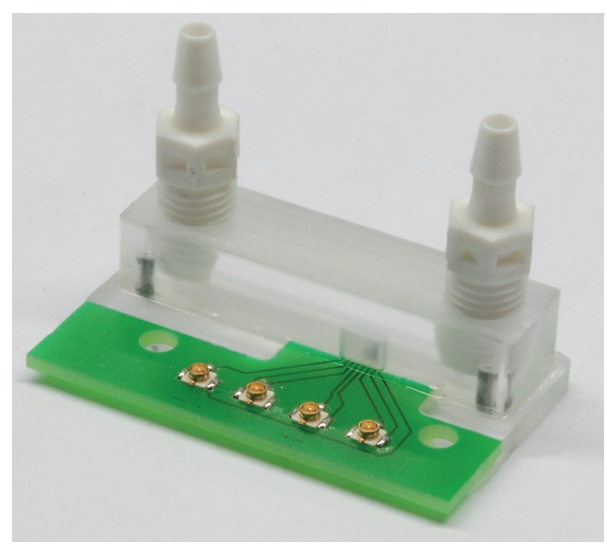

Figure 7. IMSAS flow sensor system.

of the measurement can be seen in Fig. 9. When the sensor is mounted step down, there is a point of discontinuity in the characteristic curve, comparable with the results of Eberhardt et al. (2003). Both thermopiles show this point of discontinu-

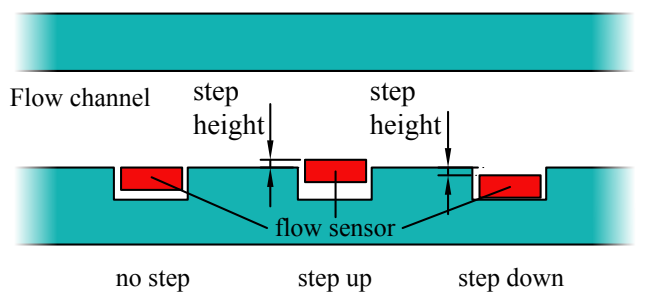

Figure 8. Flow sensor mounted in a channel in three different ways. Left: no step; middle: step up; right: step down.
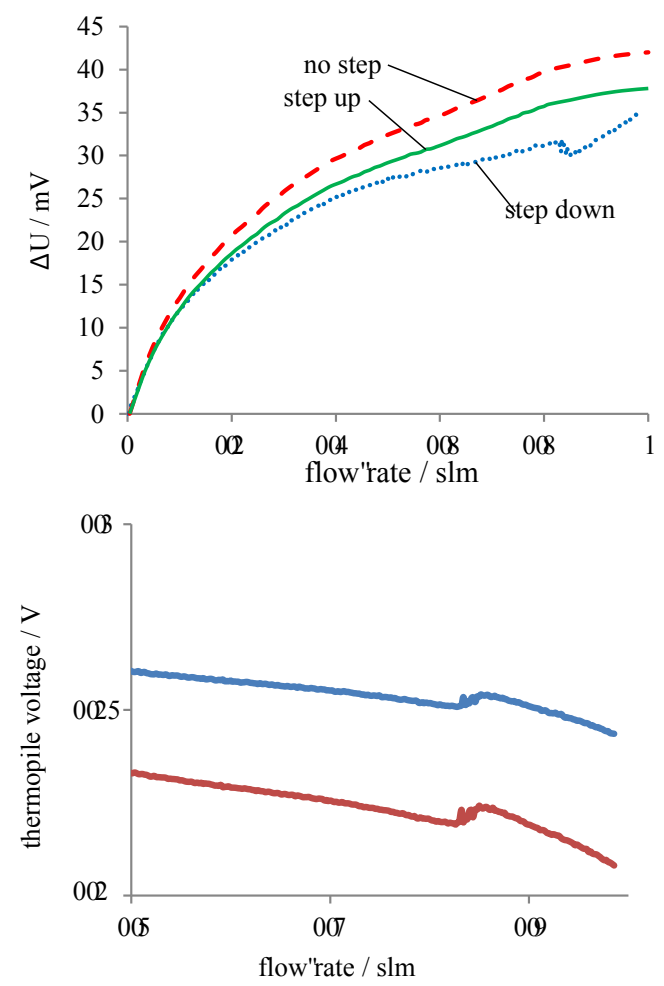

Figure 9. Top: voltage difference between the thermopiles $\Delta U$ for different step heights between the sensor and the channel wall in dependence on the flow rate $Q$ : dashed line no step, solid line step up, dotted line step down. Bottom: thermopile voltage in dependence on the flow rate for the sensor mounted step down.

ity. In contrast to the sensor mounted step down, no point of discontinuity appears in the characteristic curve of the sensor mounted step up or flush with the surface.

\section{Discussion}

Regarding the previous experimental investigations, the reason for the point of discontinuity on flow sensors mounted step down could be the change from laminar to turbulent flow. The Reynolds number for our setup is $R e=630$ for $Q=1 \mathrm{sLm}$. In addition, we calculated the standard deviation of the samples at this point to quantify the noise and thus turbulences, but the standard deviation did not change 


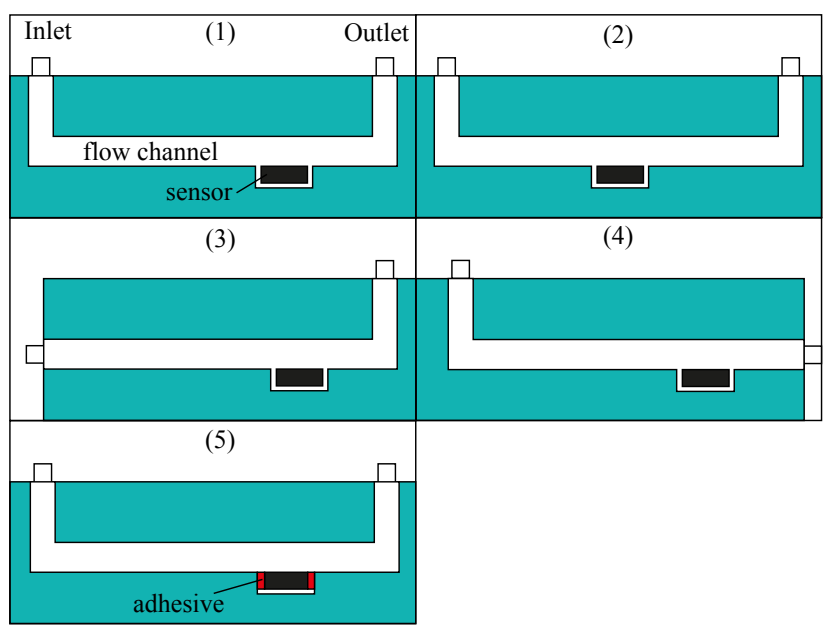

Figure 10. Different geometries of the channel: (1) rectangular inlet and outlet + sensor close to the outlet, (2) rectangular inlet and outlet + sensor in the middle of the channel, (3) straight inlet and rectangular outlet + sensor close to the outlet, (4) rectangular inlet and straight outlet + sensor close to the outlet, and (5) no pressure equalizing, fully sealing of the sensor. The sensor was always mounted flush with the surface. All measurements were also performed by changing the inlet and outlet. No points of discontinuity in the characteristic curve were measured.

in comparison to the standard deviation at lower flow rates. Thus, we can state that there is still a laminar flow. To exclude other geometry influences, we also performed different channel inlet and outlet setups as well as a fully sealed flow sensor without pressure equalizing. The measurements did not show any point of discontinuity, while they show a point of discontinuity when the sensor is mounted step down.

To investigate the flow behavior in the channel to see any causes of the point of discontinuity, we did a laminar flow simulation of the channel. The results for low and high flow rates are shown in Fig. 11. When the flow rate is low, there is flow in the cavity formed by the sensor. While increasing the flow rate, the flow in this cavity gets less, and the laminar flow profile also changes, as seen in Fig. 11 (bottom). But, a sudden change in the characteristic curve can not be explained with simple laminar flow conditions.

It is widely known that vortices can appear, although the flow is not turbulent (Tabeling, 2005; Abu-Mulaweh, 2003; Kang et al., 1999). These vortices can appear in laminar flows when there are obstacles or sudden changes in the geometry. An explanation that there is a discontinuity in the characteristic curve for a flow sensor mounted step down but not for the one mounted step up can be given by having a look at Fig. 12 . At low flow rates, no vortices appear in the cavity, as shown in the simulation in Fig. 11. At higher flow rates, vortices appear in the cavity above the sensor, influencing the thermal field and thus changing the characteristic curve, which is shown in the measurement by the point of discontinuity. For the sensor mounted step up, vortices appear before and after
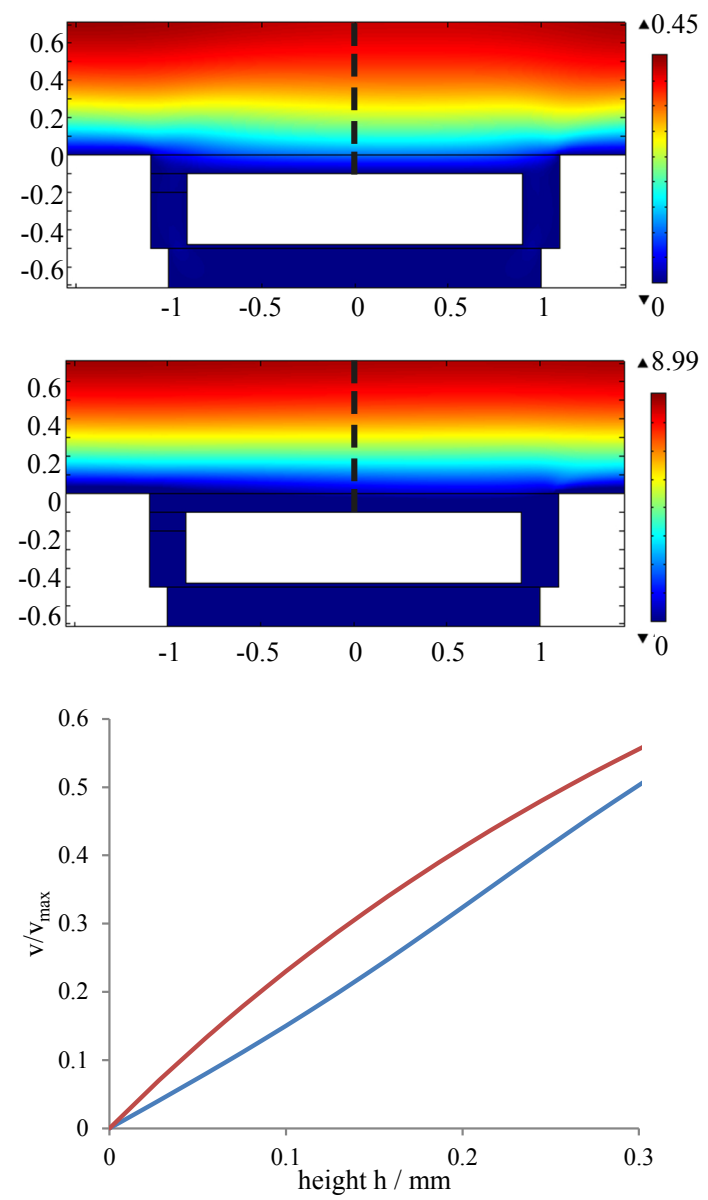

Figure 11. Top and middle: simulation of the flow velocity in the channel for flow rates 0.05 and $0.8 \mathrm{sLm}$ (COMSOL MULTIPHYSICS, laminar flow study with compressible flow). Boundary conditions: left side inlet with laminar inflow flow rate, right side $p=0$, wall $u=0$. Bottom: normalized flow velocity $v / v^{\max }$ for $0.05 \mathrm{sLm}$ (red) and $0.8 \mathrm{sLm}$ (blue) in dependence on the height of the dashed black line above the sensor.

the step (sensor) but not above the sensor. Therefore, we expect a discontinuity in the characteristic curve for step down, but not for step up. This is actually observed (Fig. 12) in different scientific papers that study a fundamental Computational Fluid Dynamics (CFD) analysis for similar geometries (Shankar, 1993; Cheng and Hung, 2006; van Dijk and de Lange, 2007). Making a CFD analysis to give a fundamental theoretical answer to what is happening in our setup when the sensor is mounted step down or step up would be rather complicated and very extensive work. It is rather complicated to simulate vortex flows, because the occurrence of vortices is dependent on the geometry, investigations into the turbulent length have to be made, and the change from laminar to turbulent flow is still part of current research and scientific papers (Cheng and Hung, 2006; Pan and Acrivos, 1967; Imberger, 1974; Shen and Floryan, 1985; Pey et al., 2014). For a flow sensor, the influence of the thermal field above the 


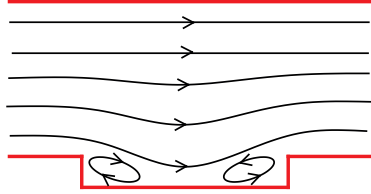

step down

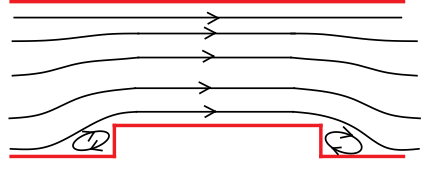

step up

Figure 12. Explanation for the discontinuity in the characteristic curve: vortices appear in the cavity and thus above the sensor mounted step down, influencing the thermal field and the characteristic curve, while vortices appear before and after but not above the sensor mounted step up.

sensor has to be taken into account too, which makes it more complicated.

So, it could be determined that a flow sensor should be mounted flush with the surface. Otherwise, a point of discontinuity in the characteristic curve can appear when the sensor is mounted step down. Mounting a sensor step up has no point of discontinuity in the characteristic curve, but it has two disadvantages: on the one hand, the cross section of the channel decreases, and on the other hand, the flow under the sensor (bypass) increases around $1.7 \%$ at $1 \mathrm{sLm}$, which results in a lower sensitivity.

\section{Conclusions}

A new packaging technology for building flow measurement systems has been presented. In the beginning, an introduction to the functional principle of flow sensors is given, and the fabrication process of our flow sensor is explained. The new packaging method was presented by describing the housing and the entire process from integrating the sensor into the bottom part to the sealing of the system. By placing a flow sensor in a channel in different step arrangements, the importance of mounting the sensor flush with the surface, in order to avoid turbulence or a reduction in the channel cross section or sensitivity, has been demonstrated. The main result of the characterization is a point of discontinuity in the characteristic curve when the sensor is mounted step down. This was analyzed by the help of theoretical investigations and scientific papers.

Edited by: R. Kirchner

Reviewed by: two anonymous referees

\section{References}

Abu-Mulaweh, H.: A review of research on laminar mixed convection flow over backward-and forward-facing steps, Int. J. Therm. Sci., 42, 897-909, 2003

Adamec, R. J. and Thiel, D. V.: Self heated thermo-resistive element hot wire anemometer, IEEE Sens. J., 10, 847-848, 2010.

Ashauer, M., Glosch, H., Hedrich, F., Hey, N., Sandmaier, H., and Lang, W.: Thermal flow sensor for liquids and gases based on combinations of two principles, Sensor. Actuat. A-Phys., 73, 713, 1999.

Billat, S., Kliche, K., Gronmaier, R., Nommensen, P., Auber, J., Hedrich, F., and Zengerle, R.: Monolithic integration of microchannel on disposable flow sensors for medical applications, Sensor. Actuat. A-Phys., 145, 66-74, 2008.

Bruschi, P., Diligenti, A., Navarrini, D., and Piotto, M.: A double heater integrated gas flow sensor with thermal feedback, Sensor. Actuat. A-Phys., 123, 210-215, 2005.

Bruschi, P., Piotto, M., and Bacci, N.: Postprocessing, readout and packaging methods for integrated gas flow sensors, Microelectr J., 40, 1300-1307, doi:10.1016/j.mejo.2008.08.009, 2009.

Buchner, R., Sosna, C., Maiwald, M., Benecke, W., and Lang, W.: A high-temperature thermopile fabrication process for thermal flow sensors, Sensor. Actuat. A-Phys., 130, 262-266, 2006.

Buchner, R., Bhargava, P., Sosna, C., Benecke, W., and Lang, W.: Thermoelectric Flow Sensors with Monolithically Integrated Channel Structures for Measurements of Very Small Flow Rates, in: IEEE Sensors, 828-831, doi:10.1109/ICSENS.2007.4388529, 2007.

Cheng, M. and Hung, K.: Vortex structure of steady flow in a rectangular cavity, Comput. Fluids, 35, 1046-1062, 2006.

Dijkstra, M., de Boer, M., Berenschot, J., Lammerink, T., Wiegerink, R., and Elwenspoek, M.: Miniaturized thermal flow sensor with planar-integrated sensor structures on semicircular surface channels, Sensor. Actuat. A-Phys., 143, 1-6, doi:10.1016/j.sna.2007.12.005, 2008.

Dillner, U., Kessler, E., Poser, S., Baier, V., and Müller, J.: Low power consumption thermal gas-flow sensor based on thermopiles of highly effective thermoelectric materials, Sensor. Actuat. A-Phys., 60, 1-4, doi:10.1016/S0924-4247(96)01409-4, 1997.

Eberhardt, W., Kück, H., Münch, M., Schilling, P., Ashauer, M., and Briegel, R.: MID-Gehäuse für ein Durchfluss-Sensorsystem, Kunststoffe, 93, 51-53, 2003.

Imberger, J.: Natural convection in a shallow cavity with differentially heated end walls. Part 3. Experimental results, J. Fluid Mech., 65, 247-260, 1974.

Ito, Y., Higuchi, T., and Takahashi, K.: Submicroscale flow sensor employing suspended hot film with carbon nanotube fins, J Therm. Sci. Tech., 5, 51-60, 2010.

Johnson, R. and Higashi, R.: A highly sensitive silicon chip microtransducer for air flow and differential pressure sensing applications, Sensor. Actuator., 11, 63-72, doi:10.1016/02506874(87)85005-9, 1987.

Kaltsas, G., Nassiopoulos, A., and Nassiopoulou, A.: Characterization of a silicon thermal gas-flow sensor with porous silicon thermal isolation, IEEE Sens. J., 2, 463-475, doi:10.1109/JSEN.2002.806209, 2002.

Kang, S., Choi, H., and Lee, S.: Laminar flow past a rotating circular cylinder, Phys. Fluids, 11, 3312, doi:10.1063/1.870190, 1999.

Liu, J., Wang, J., and Li, X.: Fully front-side bulk-micromachined single-chip micro flow sensors for bare-chip SMT (surface mounting technology) packaging, J. Micromech. Microeng., 22, 035020, doi:10.1088/0960-1317/22/3/035020, 2012.

Ma, R.-H., Wang, D.-A., Hsueh, T.-H., and Lee, C.-Y.: A MEMSbased flow rate and flow direction sensing platform with integrated temperature compensation scheme, Sensors, 9, 54605476, 2009. 
Mailly, F., Giani, A., Bonnot, R., Temple-Boyer, P., PascalDelannoy, F., Foucaran, A., and Boyer, A.: Anemometer with hot platinum thin film, Sensor. Actuat. A-Phys., 94, 32-38, 2001.

Pan, F. and Acrivos, A.: Steady flows in rectangular cavities, J. Fluid Mech., 28, 643-655, 1967.

Pey, Y. Y., Chua, L. P., and Siauw, W. L.: Effect of trailing edge ramp on cavity flow structures and pressure drag, Int. J. Heat Fluid Fl., 45, 53-71, 2014.

Sabaté, N., Santander, J., Fonseca, L., Gràcia, I., and Cané, C.: Multi-range silicon micromachined flow sensor, Sensor. Actuat. A-Phys., 110, 282-288, doi:10.1016/j.sna.2003.10.068, 2004.

Shankar, P.: The eddy structure in Stokes flow in a cavity, J. Fluid Mech., 250, 371-383, 1993.

Shen, C. and Floryan, J.: Low Reynolds number flow over cavities, Phys. Fluid., 28, 3191, doi:10.1063/1.865366, 1985.

Sosna, C., Buchner, R., Benecke, W., and Lang, W.: A Simple OneDimensional Analytical Model for Calculation of the Output Signal of Thermal Flow Sensors, in: Eurosensors XXII, Dresden, Germany, 129-132, 2008.
Sosna, C., Buchner, R., and Lang, W.: A temperature compensation circuit for thermal flow sensors operated in constanttemperature-difference mode, IEEE T. Instrum. Meas., 59, 17151721, 2010.

Sturm, H., Dumstorff, G., Busche, P., Westermann, D., and Lang, W.: Boundary Layer Separation and Reattachment Detection on Airfoils by Thermal Flow Sensors, Sensors, 12, 14292-14306, 2012.

Tabeling, P.: Introduction to Microfluidics, Oxford University Press, 2005.

van Dijk, A. and de Lange, H.: Compressible laminar flow around a wall-mounted cubic obstacle, Comput. Fluids, 36, 949-960, doi:10.1016/j.compfluid.2006.05.003, 2007.

Van Herwaarden, A. and Sarro, P.: Thermal sensors based on the Seebeck effect, Sensor. Actuator., 10, 321-346, 1986.

Van Putten, A. and Middelhoek, S.: Integrated silicon anemometer, Electron. Lett., 10, 425-426, 1974. 